\section{Autopercepção de saúde em adolescentes: prevalência e associação com fatores de risco cardiovascular}

\section{Self-rated health in adolescents: prevalence and association with cardiovascular risk factors}

Felipe Vogt Cureau ${ }^{1,2}$

Paola Marques Duarte ${ }^{1}$

Daniela Lopes Dos Santos ${ }^{3}$

Felipe Fossati Reichert ${ }^{1}$

\section{Resumo}

O objetivo deste estudo foi verificar a prevalência de autopercepção de saúde negativa e sua associação com fatores sociodemográficas e de risco cardiovascular em adolescentes. Foi realizado um estudo transversal, com amostra de 1.134 escolares, idades entre 14-19 anos, da cidade de Santa Maria, RS, Brasil. Os fatores de risco cardiovascular pesquisadas foram: inatividade física, alimentação inadequada, tabagismo, consumo excessivo de álcool, excesso de peso e pressão arterial elevada. A variável dependente investigada foi autopercepção de saúde negativa (regular/ ruim). Para análise dos fatores associados foi utilizada regressão de Poisson. A prevalência de autopercepção de saúde negativa foi de $25,7 \%$, superior nas meninas $(30,8 \%$ vs $19,9 \%$; $\mathrm{p}<0,001)$. Adolescentes com uma alimentação inadequada ( $\left.\mathrm{RP}=1,48 ; \mathrm{IC}_{950} 1,04-2,11\right)$, inativos fisicamente $\left(\mathrm{RP}=1,33 ; \mathrm{IC}_{95 \%} 1,13-1,58\right)$, fumantes ( $\left.\mathrm{RP}=1,57 ; \mathrm{IC}_{95 \%} 1,13-2,19\right)$ ou com excesso de peso $\left(\mathrm{RP}=1,76 ; \mathrm{IC}_{95 \%} 1,49-2,08\right)$ apresentaram risco para autopercepção de saúde negativa. Também foi observada uma associação linear entre o número de fatores de risco cardiovasculares e autopercepção de saúde negativa; adolescentes com quatro ou mais fatores de risco apresentaram uma razão de prevalência para autopercepção de saúde negativa de $2,73\left(\mathrm{IC}_{95 \%} 2,07-3,61\right)$ se comparado com aqueles sem fatores de risco combinados. Foi encontrada uma elevada prevalência de autopercepção de saúde negativa entre os adolescentes pesquisados. Ser do sexo feminino e apresentar fatores de risco para doenças cardiovasculares foram fatores associados à autopercepção de saúde negativa. Além disso, há uma forte associação entre o número de fatores de risco simultâneos e autopercepção de saúde negativa.

\section{Palavras-chave}

Saúde do adolescente, Estilo de vida, Escolares, Estudos transversais.

\begin{abstract}
The aim this study was to determine the prevalence of negative self-rated health and the association with sociodemographic variables and cardiovascular risk factors in adolescents. We conduct a cross sectional study, with 1,134 high school adolescents, aged between 14-19 years old, from the city of Santa Maria, RS, Brazil. Cardiovascular risk factors studied were: physical inactivity, unhealthy diet, smoking, excessive alcohol consumption, overweight and high blood pressure. The dependent variable investigated was negative self-rated health. Correlates were investigated using Poisson regression. The prevalence of poorer self-rated health was $25.7 \%$ and it was higher among girls (30.8\% vs $19.9 \%$, $p<0.001)$. Adolescents with an inadequate diet (PR=1.48; 95\%CI 1.04-2.11), physically inactive $(P R=1.33 ; 95 \% C I$ 1.13-1.58), that smoked $(P R=1.57 ; 95 \% C I$ 1.13-2.19) or were overweight $(P R=1.76 ; 95 \% C I 1.49-2.08)$ presented a risk of negative self-rated health. A linear association was observed between the number of risk factors and poorer self-rated health. Adolescents with four or more simultaneous risk factors had a prevalence ratio for negative self-perceived health of 2.73 (95\%CI 2.07-3.61). A high prevalence of poorer self-rated health among the surveyed adolescents was found and female sex as well as the presence of cardiovascular risk factors, were factors associated with that condition. Furthermore, there is a linear relationship between the numbers of risk factors in cluster and negative self-perceived health.
\end{abstract}

\section{Keywords}

Adolescent health, Life style, Students, Cross-sectional studies.
Rev Bras Ativ Fis Saúde p. 750-760 $\mathrm{DOI}$

http://dx.doi.org/10.12820/rbafs.v.18n6p750

1 Universidade Federal de Pelotas, Programa de Pós-Graduação em Educação Física, Pelotas, Rio Grande do Sul, Brasil.

2 Universidade Federal do Rio Grande do Sul, Programa de Pós-Graduação em Endocrinologia, Porto Alegre, Rio Grande do Sul, Brasil.

3 Universidade Federal de Santa Maria, Programa de Pós-Graduação em Educação Física, Santa Maria, Rio Grande do Sul, Brasil. 


\section{INTRODUÇÃO}

A autopercepção de saúde (APS) é um importante indicador global de saúde. A sua facilidade de obtenção, muitas vezes avaliada por meio de uma única questão, ${ }^{1-3}$ e sua relevância, seja pela informação obtida em si ou através da associação com diferentes desfechos em saúde, ${ }^{4,5}$ fazem com que essa variável receba cada vez mais atenção em estudos epidemiológicos.

Uma APS negativa entre indivíduos jovens é um importante preditor de mortalidade, representado um risco relativo de $4,5\left(\mathrm{IC}_{95 \%} 1,1-18,7\right)$ e $10,3\left(\mathrm{IC}_{95 \%} 6,3-\right.$ 16,9) para indivíduos nas faixas etárias de 16-24 e 25-34 anos, respectivamente, se comparado àqueles que apresentam uma APS positiva. ${ }^{4}$ Além disso, a APS negativa é um preditor independente para morbidades como, por exemplo, alterações em marcadores inflamatórios de doença cardiovascular ${ }^{5}$ e doença coronariana. ${ }^{6}$

Em adolescentes, a prevalência de APS negativa (regular/ruim) é de aproximadamente $15 \%,{ }^{1,2,7}$ estando maiores percentuais associados ao sexo feminino, pior condição econômica e presença de fatores de risco como, por exemplo, obesidade e tabagismo. ${ }^{1,2}$ A presença de fatores de risco para doenças cardiovascular em adolescentes está associada a modificações na saúde física de adolescentes e eleva o risco de morbidades na idade adulta. ${ }^{8}$ No entanto, a relação desses fatores de risco com a saúde percebida de adolescentes é um tema pouco abordado.

Portanto, o objetivo deste estudo foi verificar a prevalência de APS negativa e sua possível associação com variáveis sociodemográficas e fatores de risco para doenças cardiovasculares, individuais e simultâneos, em adolescentes de uma cidade dos sul do Brasil.

\section{MÉTODOS}

\section{População e amostra}

Este estudo transversal foi realizado na cidade de Santa Maria, localizada na região central do Rio Grande do Sul. De acordo com o censo de 2010 a cidade é a quinta mais populosa do estado (261.031 habitantes). Cerca de 20.000 habitantes da cidade estão na faixa etária de 15-19 anos de idade. A população alvo do estudo foi composta por adolescentes escolares (14-19 anos), matriculados no período diurno, das 35 escolas de ensino médio existentes na cidade.

Inicialmente o tamanho de amostra foi calculado para atender ao objetivo de um estudo para verificar a prevalência e fatores associados à ocorrência simultânea de fatores de risco para doenças crônicas em adolescentes, sendo o presente estudo uma análise secundária de dados de um projeto com objetivo mais amplo. 9, 10 Para os objetivos do presente estudo o cálculo da precisão estatística foi realizado a posteriori. A amostra analisada $(\mathrm{n}=1.134)$ permitiu estimar uma prevalência de autopercepção de saúde regular/ruim de $25 \%$ com erro de 2,5 pontos percentuais e nível de confiança de $95 \%$. Para as medidas de associação a amostra tem poder de $80 \%$ e nível de confiança de $95 \%$ para detectar razões de prevalência iguais ou superiores a 1,35 como significativas $(\mathrm{p}<0,05)$.

A amostragem foi realizada em dois estágios: seleção de escolas e de turmas. Primeiramente, solicitou-se a todas as escolas de ensino médio ( $n=35)$ uma listagem com o número de turmas e a distribuição dessas quanto à série. Com posse dessas informações, todas as turmas de ensino médio da cidade $(n=300)$ foram listadas em uma planilha eletrônica. As turmas da mesma escola foram ordenadas por série. Logo após, os blocos de turmas de cada escola foram estratificados por 
rede de ensino (estadual, federal e particular), região da cidade (centro, norte, sul, leste e oeste) e ordenados de forma decrescente quanto ao número de turmas na escola (porte).

Após organizar a planilha, empregou-se uma estratégia sistemática de amostragem. Para tanto, estimou-se que, em média, cada turma tivesse 25 alunos e com a necessidade de estudar $~ 1.200$ adolescentes, foi selecionada uma a cada seis turmas. Isso determinou que 50 turmas fossem estudadas, as quais foram distribuídas em 20 escolas (14 estaduais, 4 particulares e 2 federais). Em cada escola foram pesquisadas entre uma e oito turmas, distribuição proporcional ao porte da instituição de ensino. Apenas alunos do período diurno foram elegíveis para o estudo, pois, de acordo com levantamento feito junto as escolas havia um baixo percentual de adolescentes ( $\leq 19$ anos) no ensino noturno, além das dificuldades logísticas que representaria a coleta de dados no turno da noite, então optou-se por não incluir estudantes desse turno na amostra.

\section{Coleta de dados}

O trabalho de campo estendeu-se de abril a agosto de 2011. A equipe de pesquisa foi composta por seis profissionais de Educação Física, treinados para aplicação do questionário, realização das medidas antropométricas e verificação da pressão arterial.

\section{Variável dependente}

A APS dos adolescentes foi investigada por meio da seguinte pergunta: como você considera sua saúde?1,2,11 Com as seguintes opções de resposta: excelente, muito boa, boa, regular ou ruim. Para fins de análise as alternativas foram categorizadas de forma dicotômica: positiva (excelente/muito boa/boa) e negativa (regular/ruim).

\section{Variáveis independentes}

Os indicadores sociodemográficos pesquisados foram sexo; idade, coletada a partir da data de nascimento e depois categorizada (14-15; 16-17; 18-19 anos); cor da pele (branca ou não-branca); rede de ensino (pública ou privada); e condição econômica, determinada por meio do Critério Brasil (http://www.abep.org), índice que leva em consideração a posse de bens no domicílio, presença de empregada doméstica e a escolaridade do chefe da família. O escore obtido por meio da aplicação do Critério Brasil foi posteriormente categorizado em tercis.

Também foram pesquisados os seguintes fatores de risco para doenças cardiovasculares: inatividade física, alimentação inadequada, tabagismo, consumo excessivo de álcool, excesso de peso e pressão arterial elevada. Para determinação do nível de atividade física foi utilizado um questionário que avaliou atividades físicas no lazer e no deslocamento para a escola durante a última semana. ${ }^{12}$ Foi considerando inativo o adolescente que não acumulou, pelo menos, 300 minutos/semana em atividades físicas moderadas a vigorosas. ${ }^{13}$

Os hábitos alimentares foram coletados por meio do questionário de frequência alimentar proposto por Block, ${ }^{14}$ o qual investiga o consumo de gorduras e fibras durante o último ano. Adolescentes com 27 pontos ou mais no escore do consumo de gorduras ou 20 pontos ou menos no escore de fibras foram considerados com alimentação inadequada. ${ }^{14}$

Foram considerados fumantes aqueles que referiram ter consumido cigarros, independente da quantidade, nos últimos 30 dias. Os adolescentes que referiram ter ingerido cinco ou mais doses de álcool em pelo menos uma ocasião do último mês foram classificado como tendo consumido álcool em excesso. 
O excesso de peso foi determinado pelo índice de massa corporal (IMC), calculado pela divisão da massa corporal pelo quadrado da estatura, com base nos valores de referência propostos por Cole et al. ${ }^{15} \mathrm{~A}$ massa corporal foi mensurada por uma balança digital com precisão de $0,1 \mathrm{~kg}$, estando o avaliado descalço e com roupas leves. A estatura foi mensurada com auxílio de um estadiômetro com precisão de $0,1 \mathrm{~cm}$, estando o adolescentes descalço e livre de qualquer acessório que pudesse interferir na medida.

A verificação da pressão arterial foi feita com o monitor OMRON HEM 742, validado para uso em adolescentes brasileiros. ${ }^{16}$ Todas as recomendações da VI Diretriz Brasileira de Hipertensão Arterial ${ }^{17}$ foram respeitadas. A medida foi realizada duas vezes, no braço direito dos adolescentes, com cinco minutos de intervalo entre elas. A média de duas medidas foi utilizada nas análises. Não foram realizadas medidas de pressão arterial após provas ou aulas de Educação Física, pois, tanto o estresse físico como mental podem influenciar a medida em repouso, elevando, principalmente, o número de casos falso-positivos. Adolescentes com pressão arterial acima do percentil 90 para sexo, idade e estatura foram considerados com pressão arterial elevada. ${ }^{18}$ Para indivíduos com idade a partir de 18 anos foi utilizado o ponto de corte de $130 / 85 \mathrm{mmHg}$ para indicar pressão arterial elevada. ${ }^{17}$

\section{Análise estatística}

Os dados foram digitados com dupla entrada no Epi-Info 6.04, e a análise de dados foi conduzida no STATA 12.0. A análise descritiva envolveu medidas absolutas e relativas. A associação entre a prevalência de APS negativa e as variáveis independentes foi verificada pelo teste exato de Fischer ou qui-quadrado para tendência linear.

As razões de prevalência (RP) bruta e ajustada foram calculadas por regressão de Poisson. $\mathrm{Na}$ análise multivariável foi utilizado um modelo hierárquico com as variáveis sociodemográficas no nível distal e fatores de risco no nível proximal. O ajuste do modelo deu-se entre as variáveis de mesmo nível e aquelas de nível mais distal, sendo mantidas no modelo as variáveis com valor $\mathrm{p}<0,2$.

Além da análise da associação entre os fatores de risco individuais e APS negativa, foi criado um escore de simultaneidade de fatores de risco, no qual foi atribuído um ponto a cada fator de risco e feito um somatório simples da pontuação (variação do escore: $0=$ nenhum fator de risco; $6=$ todos). As RP envolvendo a ocorrência simultânea de fatores de risco e APS negativa foram ajustadas para as variáveis sociodemográficas pesquisadas.

O conjunto de comandos "survey" foi utilizado para consideração de efeito do delineamento sobre as análises. Neste estudo foram considerados significativos valores de $\mathrm{p}<0,05$.

\section{Aspectos éticos}

Este estudo foi a provado pelo Comitê de Ética em Pesquisa com Seres Humanos da Universidade Federal de Santa Maria. A participação dos adolescentes no estudo foi condicionada a entrega do Termo de Consentimento Livre e Esclarecido, assinado pelos pais ou responsável.

\section{RESULTADOS}

Ao todo, 1.376 adolescentes foram elegíveis e 1.134 participaram da pesquisa. Houve 161 perdas (adolescentes ausentes no momento da coleta), 71 recusas e 10 exclusões por preenchimento inadequado do questionário. A pressão arterial foi 
a variável com maior número de perdas, sendo avaliada em 1.064 adolescentes. A tabela 1 apresenta características descritivas da amostra. A média de idade na amostra foi de $16,3(\mathrm{DP} \pm 1,1)$ anos, com maior percentual de meninas $(53,9 \%)$, adolescentes de cor da pele branca $(73,7 \%)$ e estudantes de escolas públicas $(77,8)$.

A figura 1 apresenta a distribuição, estratificada por sexo, das respostas para APS nas cinco categorias possíveis. A prevalência, para o total da amostra, de APS negativa foi de $25,7 \%$ ( $\mathrm{IC}_{95 \%} 23,2 \%-28,3 \%$ ), sendo superior entre as meninas $(30,8 \%$ vs $19,9 \% ; \mathrm{p}<0,001)$ se comparado aos meninos, e na presença de alguns fatores de risco para doenças crônicas (tabela 1).

Tabela 1 - Composição da amostra e prevalência de autopercepção de saúde negativa em adolescentes.

\begin{tabular}{|c|c|c|c|}
\hline Variáveis & $\mathrm{N}(\%)$ da amostra & $\begin{array}{l}\text { Autopercepção de } \\
\text { saúde negativa (\%) }\end{array}$ & Valor-p \\
\hline \multicolumn{4}{|l|}{ SOCIODEMOGRÁFICAS } \\
\hline Sexo & & & $<0,001^{*}$ \\
\hline Masculino & $523(46,1)$ & 19,9 & \\
\hline Feminino & $611(53,9)$ & 30,8 & \\
\hline Idade (anos) & & & $0,866^{* *}$ \\
\hline $14-15$ & $415(36,6)$ & 25,3 & \\
\hline $16-17$ & $644(56,8)$ & 26,5 & \\
\hline $18-19$ & $75(6,6)$ & 21,3 & \\
\hline Cor da pele & & & $0,426^{*}$ \\
\hline Branca & $796(73,7)$ & 25,7 & \\
\hline Não-branca & $284(26,3)$ & 23,2 & \\
\hline Nível econômico (tercil) & & & $0,131^{* *}$ \\
\hline $1^{\circ}$ (mais ricos) & $312(29,0)$ & 22,8 & \\
\hline $2^{\circ}$ & $359(33,4)$ & 24,8 & \\
\hline $3^{\circ}$ (mais pobres) & $405(37,6)$ & 27,6 & \\
\hline Rede de ensino & & & $0,253^{*}$ \\
\hline Pública & $882(77,8)$ & 24,9 & \\
\hline Privada & $252(22,2)$ & 28,6 & \\
\hline \multicolumn{4}{|c|}{ FATORES DE RISCO CARDIOVASCULAR } \\
\hline Alimentação inadequada & & & $0,029^{*}$ \\
\hline Não & $155(14,1)$ & 18,7 & \\
\hline Sim & $945(85,9)$ & 27,1 & \\
\hline Inatividade física & & & $0,001^{*}$ \\
\hline Não & $530(46,7)$ & 20,9 & \\
\hline Sim & $604(53,3)$ & 30,0 & \\
\hline Fumante & & & $0,001^{*}$ \\
\hline Não & $1.035(91,4)$ & 24,4 & \\
\hline Sim & $97(8,6)$ & 40,2 & \\
\hline Consumo excessivo de álcool & & & $0,221^{*}$ \\
\hline Não & $875(77,6)$ & 25,0 & \\
\hline Sim & $252(22,4)$ & 29,0 & \\
\hline Excesso de peso & & & $<0,001^{*}$ \\
\hline Não & $843(76,1)$ & 22,4 & \\
\hline Sim & $264(23,9)$ & 36,4 & \\
\hline Pressão arterial elevada & & & $0,819^{*}$ \\
\hline Não & $733(68,9)$ & 25,6 & \\
\hline Sim & $331(31,1)$ & 24,8 & \\
\hline
\end{tabular}

* Teste exato de Fisher para heterogeneidade; ${ }^{* *}$ Teste do Qui-quadrado para tendência linear. 


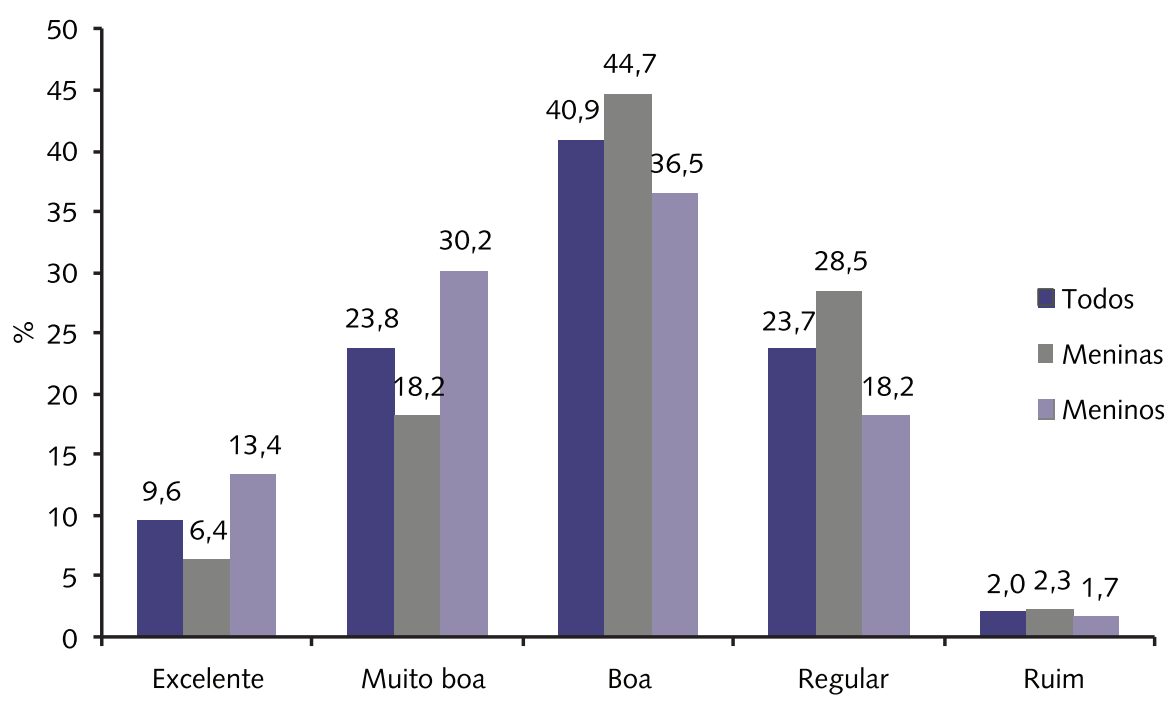

Figura 1 - Descrição da autopercepção de saúde em adolescentes.

A tabela 2 apresenta a associação bruta e ajustada entre APS regular/ruim e as variáveis independentes. As razões de prevalência ajustadas evidenciaram que as meninas apresentam um risco $58 \%\left(\mathrm{RP}=1,58 ; \mathrm{IC}_{95 \%} 1,27-1,98\right)$ maior de perceberem a saúde como regular/ruim se comparado aos meninos, sendo a única das variáveis sociodemográficas associada ao desfecho.

Tabela 2 - Razões de prevalência (RP) bruta e a ajustada para associação entre autopercepção de saúde negativa e variáveis independentes em adolescentes.

\begin{tabular}{|c|c|c|c|c|}
\hline \multirow[b]{2}{*}{ Variáveis } & \multicolumn{4}{|c|}{ Autopercepção de saúde negativa } \\
\hline & RP bruta $\left(\mathrm{IC}_{95 \%}\right)$ & Valor-p & $\begin{array}{l}\text { RP ajustada } \\
\left(\mathrm{IC}_{95 \%}\right)\end{array}$ & Valor-p \\
\hline \multicolumn{5}{|l|}{ SOCIODEMOGRÁFICAS } \\
\hline Sexo & & $<0,001^{*}$ & & $<0,001^{*}$ \\
\hline Masculino & 1 & & 1 & \\
\hline Feminino & $1,55(1,24-1,92)$ & & $1,58(1,27-1,98)$ & \\
\hline Idade (anos) & & $0,889^{* *}$ & & $0,868^{* *}$ \\
\hline $14-15$ & 1 & & 1 & \\
\hline $16-17$ & $1,05(0,82-1,35)$ & & $1,07(0,82-1,41)$ & \\
\hline $18-19$ & $0,84(0,47-1,52)$ & & $0,78(0,40-1,54)$ & \\
\hline Cor da pele & & $0,385^{*}$ & & $0,450^{*}$ \\
\hline Branca & 1 & & 1 & \\
\hline Não-branca & $0,90(0,71-1,15)$ & & $0,92(0,74-1,15)$ & \\
\hline Nível econômico (tercil) & & $0,104^{* *}$ & & $0,073^{* *}$ \\
\hline $1^{\circ}$ (mais ricos) & 1 & & 1 & \\
\hline $2^{\circ}$ & $1,09(0,79-1,50)$ & & $1,14(0,84-1,53)$ & \\
\hline $3^{\circ}$ (mais pobres) & $1,22(0,95-1,55)$ & & $1,25(0,97-1,60)$ & \\
\hline Rede de ensino & & $0,193^{*}$ & & $0,070^{*}$ \\
\hline Pública & 1 & & 1 & \\
\hline Privada & $1,15(0,93-1,41)$ & & $1,21(0,98-1,50)$ & \\
\hline \multicolumn{5}{|c|}{ FATORES DE RISCO CARDIOVASCULAR } \\
\hline Alimentação inadequada & & $0,025^{*}$ & & $0,032^{*}$ \\
\hline Não & 1 & & 1 & \\
\hline Sim & $1,45(1,05-1,99)$ & & $1,48(1,04-2,11)$ & \\
\hline Inatividade física & & $<0,001^{*}$ & & $0,002^{*}$ \\
\hline
\end{tabular}




\begin{tabular}{|c|c|c|c|c|}
\hline \multirow[b]{2}{*}{ Variáveis } & \multicolumn{4}{|c|}{ Autopercepção de saúde negativa } \\
\hline & RP bruta $\left(I C_{95 \%}\right)$ & Valor-p & $\begin{array}{c}\text { RP ajustada } \\
\left(\mathrm{IC}_{95 \%}\right)\end{array}$ & Valor-p \\
\hline Não & 1 & & 1 & \\
\hline Sim & $1,43(1,20-1,70)$ & & $1,33(1,13-1,58)$ & \\
\hline Fumante & & $<0,001^{*}$ & & $0,010^{*}$ \\
\hline Não & 1 & & 1 & \\
\hline Sim & $1,64(1,28-2,11)$ & & $1,57(1,13-2,19)$ & \\
\hline Consumo excessivo de álcool & & $0,152^{*}$ & & $0,848^{*}$ \\
\hline Não & 1 & & 1 & \\
\hline Sim & $1,16(0,94-1,42)$ & & $0,97(0,71-1,33)$ & \\
\hline Excesso de peso & & $<0,001^{*}$ & & $<0,001^{*}$ \\
\hline Não & 1 & & 1 & \\
\hline Sim & $1,62(1,33-1,98)$ & & $1,76(1,49-2,08)$ & \\
\hline Pressão arterial elevada & & $0,796^{*}$ & & $0,944^{*}$ \\
\hline Não & 1 & & 1 & \\
\hline Sim & $0,97(0,73-1,27)$ & & $1,01(0,79-1,29)$ & \\
\hline
\end{tabular}

* Teste de Wald para heterogeneidade; ${ }^{* *}$ Teste de Wald para tendência linear;

A análise multivariável também demonstrou que adolescentes com uma alimentação inadequada $\left(\mathrm{RP}=1,48 ; \mathrm{IC}_{95 \%} 1,04-2,11\right)$, inativos fisicamente $(\mathrm{RP}=1,33$; $\left.\mathrm{IC}_{95 \%} 1,13-1,58\right)$, fumantes $\left(\mathrm{RP}=1,57 ; \mathrm{IC}_{95 \%} 1,13-2,19\right)$ ou com excesso de peso $\left(\mathrm{RP}=1,76 ; \mathrm{IC}_{95 \%}, 1,49-2,08\right)$ apresentam risco para APS negativa.

A tabela 3 apresenta uma associação entre o número de fatores de risco e APS negativa. Quanto maior o número de fatores de risco simultâneos, maior a RP para APS regular/ruim. Essa associação se manteve significativa após ajuste para variáveis sociodemográficas, quando, por exemplo, adolescentes com quatro ou mais fatores de risco apresentaram uma RP para APS negativa de 2,73 $\left(\mathrm{IC}_{95 \%} 2,07-3,61\right)$ se comparados aos adolescentes que não apresentaram fatores de risco combinados.

Tabela 3 - Razões de prevalência (RP) bruta e a ajustada para associação entre a ocorrência simultânea de fatores de risco cardiovascular e autopercepção de saúde negativa em adolescentes.

\begin{tabular}{lcccc}
\hline \multirow{2}{*}{ Variável } & \multicolumn{3}{c}{ Autopercepção de saúde negativa } \\
\cline { 2 - 5 } & RP bruta $\left(I C_{95 \%}\right)$ & Valor-p & RP ajustada $\left(I C_{95 \%}\right)$ & Valor-p \\
\hline Número de fatores de risco & & $<0,001^{*}$ & $<0,001^{*}$ \\
\hline 0 ou 1 & 1 & $1,43(1,11-1,86)$ & \\
\hline 2 & $1,47(1,15-1,87)$ & $1,67(1,25-2,24)$ & \\
\hline 3 & $1,57(1,19-2,07)$ & $2,73(2,07-3,61)$ & \\
\hline 4 ou mais & $2,58(1,97-3,37)$ & & 1 & \\
\hline
\end{tabular}

* Teste de Wald para tendência linear.

\section{DISCUSSÃO}

Este estudo teve como objetivo investigar a prevalência e fatores associados a APS negativa em adolescentes. Seus resultados apontam que um em cada quatro adolescentes pesquisados referiram perceber a saúde como regular/ruim. Ser do sexo feminino apresentou associação direta com o desfecho pesquisado, assim como uma alimentação inadequada, inatividade física, tabagismo e excesso de peso. Além disso, encontrou-se uma forte associação entre o número de fatores de risco simultâneos e o relato de APS negativa entre os adolescentes. 
A prevalência encontrada de 25,7\% para APS negativa neste estudo é superior à reportada por outras pesquisas envolvendo adolescentes, ${ }^{1-3,11}$ sendo semelhante à encontrada em adultos $(22,3 \%)$ e menor em relação a idosos $(49,4 \%)$ da cidade de Pelotas-RS. ${ }^{11} \mathrm{O}$ estudo Health Behaviour in School-Aged Children, ${ }^{7}$ que engloba 41 países encontrou uma prevalência global de APS negativa de $18 \%$ aos 15 anos de idade, com amplitude de 6\% na Macedônia a 37\% na Ucrânia. No Brasil, estudos apontam uma variação na prevalência de APS negativa de $12,1 \%{ }^{11} \mathrm{em}$ adolescentes de Pelotas-RS, a 24,4\% em adolescentes de Ceilândia-DF. ${ }^{19}$

Os estudos com adolescentes, em sua maioria, apontam maior prevalência de APS negativa entre as meninas ${ }^{1-3}$. Uma explicação para isso pode ser o fato das meninas serem mais atentas, sensíveis e melhor informadas em relação à saúde, percebendo-a de maneira global, considerando aspectos biológicos, psicológicos e sociais. ${ }^{1}$ Além disso, a procura por consultas médicas é $50 \%$ maior entre as mulheres se comparado aos homens,$^{20}$ as consultas de rotina iniciadas ainda na adolescência pelas meninas podem levar ao diagnóstico precoce de alguma doença, e implicar de forma direta na APS, ao contrário dos meninos que dificilmente realizam consultas preventivas.

Entre os fatores de risco pesquisados apenas a pressão arterial elevada e consumo excessivo de álcool não apresentaram associação com a APS negativa. Em relação à pressão arterial elevada é importante destacar que se trata de um problema assintomático e que os adolescentes não costumam avaliar os níveis pressóricos de forma preventiva. ${ }^{21}$

O consumo de álcool entre adolescentes além de frequente é muitas vezes associado a questões positivas como desinibição e melhora nas relações sociais. ${ }^{7}$ Além disso, a informação de que o consumo moderado de álcool pode ser benéfico a saúde aliado ao consumo no ambiente familiar podem justificar a não percepção dos adolescentes do consumo de álcool como um fator de risco para diversas doenças.

Neste estudo o índice alimentar utilizado levou em consideração apenas a frequência de consumo de fibras e gorduras. Estudo envolvendo adolescentes americanos utilizou um índice alimentar com maior número de nutrientes, mas apenas o consumo de vegetais e de gorduras esteve associado à APS. ${ }^{22}$ Entre adolescentes brasileiros, mais de $80 \%$ têm uma dieta pobre em fibras e um terço consome gordura em excesso. ${ }^{23}$ Esses dados indicam que tais nutrientes devem ser objeto de estudo de futuras intervenções alimentares em adolescentes, pois afetam indicadores clínicos e percebidos de saúde.

O estudo da associação entre atividade física e APS em adolescentes é algo recente, porém, resultados de uma revisão sistemática sobre o tema que compilou 16 estudos, a maioria com delineamento transversal, aponta que há uma relação direta entre as variáveis. ${ }^{24}$ Além disso, estudos longitudinais com adolescentes reforçam essa relação, pois menores níveis de atividade física são determinantes da APS negativa durante a adolescência, ${ }^{25}$ relação que é mais forte entre os meninos. ${ }^{26}$ Atividades simples, como a caminhada, favorecem uma APS positiva, ${ }^{27}$ porém os melhores resultados estão associados com atividades físicas realizadas com alta frequência e intensidade. ${ }^{28}$

Tabagismo e excesso de peso também foram associados a uma APS negativa, fato observado em estudos anteriores. ${ }^{1-3}$ A relação com o tabagismo pode ser explicada, ao menos em parte, pela maciça divulgação dos males associados a esse comportamento. ${ }^{29} \mathrm{O}$ excesso de peso, por sua vez, além dos diversos riscos que representa a saúde, não é parte do estereótipo de imagem corporal desejado pelos adolescentes, hipótese que pode ligá-lo a APS negativa. ${ }^{30}$ 
Além de evidenciar a associação de alguns fatores de risco individuais com APS negativa em adolescentes, este estudo demonstra que há uma associação entre aumento no número de fatores de risco simultâneos e APS negativa. Uma interpretação plausível para esta associação é que os adolescentes conseguem identificar como prejudicial à saúde a presença desses fatores de risco, hipótese que deve ser investigada no futuro.

Este estudo apresenta algumas limitações que precisam ser consideradas. Não é possível afastar a possibilidade de viés de causalidade reversa entre os fatores de risco estudados e APS, uma vez que ambos foram coletados em um mesmo momento, ou seja não é possível indicar uma relação temporal entre as varáveis ou inferir causalidade. Além disso, não é possível descartar um viés de seleção, pois adolescentes que estavam fora das escolas ou estudavam no período noturno não fizeram parte da amostra. Suspeita-se que isso possa ter subestimado tanto a prevalência de APS negativa, quanto sua força de associação com fatores de risco para doenças cardiovasculares. Portanto, a composição da amostra permite a extrapolação adequada dos resultados apenas para adolescentes escolares do período diurno.

Como pontos fortes do estudo, ressalta-se que foram incluídos adolescentes de ambos os sexos, diferentes idades e de todas as redes de ensino com nível médio da cidade, bem como distribuídos por todas as regiões. Além disso, destaca-se que este estudo investigou alguns dos principais fatores de risco para doenças cardiovasculares, sendo, até onde se tem conhecimento, o primeiro a investigar a relação entre a ocorrência simultânea desses fatores com a APS negativa em adolescentes.

\section{CONCLUSÃO}

Entre os adolescentes pesquisados 25,7\% referiram uma APS negativa, prevalência elevada para a faixa etária estudada. Ser do sexo feminino e apresentar fatores de risco para doenças cardiovasculares foram fatores de risco para ocorrência de APS negativa. Além disso, a medida que há aumento no número de fatores de risco simultâneos, também eleva-se o risco de APS negativa.

Os resultados encontrados reforçam a importância e a validade da medida da APS na faixa etária estudada. A medida apesar de simples é um indicador eficaz para avaliar a saúde física e percebida, pois está fortemente associada a fatores de risco comportamentais e biológicos em adolescentes.

\section{Agradecimentos}

A Coordenação de Aperfeiçoamento de Pessoal de Nível Superior (CAPES), pela bolsa de estudos concedida a Felipe Vogt Cureau. A Fundação de Amparo à Pesquisa do Estado do Rio Grande (FAPERGS) pela bolsa de estudos concedida a Paola Marques Duarte.

\section{Contribuição dos autores}

Felipe V. Cureau e Felipe F Reichert participaram de todas as fases do estudo, desde a concepção do projeto de pesquisa à redação do artigo. Paola M. Duarte e Daniela Lopes dos Santos participaram da elaboração e condução do trabalho de campo e da escrita do artigo.

\section{REFERÊNCIAS}

1. Mendonça G, Farias Júnior JC. Percepção de saúde e fatores associados em adolescentes. Rev Bras Ativ Fis e Saúde 2012;17(3):174-180. 
2. Sousa TF, Silva KS, Garcia LMT, Del Duca GF, Oliveira ESA, Nahas MV. Autoavaliação de saúde e fatores associados em adolescentes do Estado de Santa Catarina, Brasil. Rev Paul Pediatr 2010;28(4):333-9.

3. Foti K, Eaton D. Associations of selected health risk behaviors with self-rated health status among U.S. high school students. Public Health Rep 2010;125(5):771-81.

4. Burstrom B, Fredlund P. Self rated health: Is it as good a predictor of subsequent mortality among adults in lower as well as in higher social classes? J Epidemiol Community Health 2001;55(11):836-40.

5. Christian LM, Glaser R, Porter K, Malarkey WB, Beversdorf D, Kiecolt-Glaser JK. Poorer self-rated health is associated with elevated inflammatory markers among older adults. Psychoneuroendocrinology 2011;36(10):1495-504.

6. Moller L, Kristensen TS, Hollnagel H. Self rated health as a predictor of coronary heart disease in Copenhagen, Denmark. J Epidemiol Community Health 1996;50(4):423-8.

7. World Health Organization. Health behavior in school-aged children: international report from the 2005/2006 survey. Scotland 2008.

8. Berenson GS. Childhood risk factors predict adult risk associated with subclinical cardiovascular disease. The Bogalusa Heart Study. Am J Cardiol 2002;90(10C):3L-7L.

9. Cureau FV, Duarte P, Santos DL, Reichert FF. Clustering of Risk Factors for NonCommunicable Diseases in Brazilian Adolescents: Prevalence and Correlates. J Phys Act Health (in press) 2014;11(5).

10. Cureau FV, Reichert FF. Indicadores antropométricos de obesidade como preditores de pressão arterial elevada. Rev Bras Cineantropom Desempenho Hum 2013;15(3).

11. Reichert FF, Loch MR, Capilheira MF. [Self-reported health status in adolescents, adults and the elderly]. Cien Saude Colet 2012;17(12):3353-62.

12. de Farias Júnior JC, Lopes AS, Mota J, Santos MP, Ribeiro JC, Hallal PC. Validade e reprodutibilidade de um questionário para medida de atividade física em adolescentes. Rev. bras. epidemiol 2011;15(1):198-210.

13. World Health Organization. Global Recommendations on Physical Activity for Health. Geneve 2010.

14. Thompson FE, Byers T. Dietary assessment resource manual.J Nutr 1994;124(11 Suppl):2245S2317S.

15. Cole TJ, Bellizzi MC, Flegal KM, Dietz WH. Establishing a standard definition for child overweight and obesity worldwide: international survey. BMJ 2000;320(7244):1240-3.

16. Christofaro DG, Fernandes RA, Gerage AM, Alves MJ, Polito MD, Oliveira AR. Validation of the Omron HEM 742 blood pressure monitoring device in adolescents. Arq Bras Cardiol 2009;92(1):10-5.

17. Nobre F, Brandão AA, Rodrigues CIS, Consolim-Colombo F, Plavik FL, Malachias MVB, et al. VI Diretrizes Brasileiras de Hipertensão. Rev Bras Hipertens 2010;17(1):1-64.

18. National high blood pressure education program working group on hypertension control in children and adolescents. The fourth report on the diagnosis, evaluation, and treatment of high blood pressure in children and adolescents. Pediatrics 2004;114(2 Suppl.):555-576.

19. Souza EM, Abrão MPS, Motta IA, Almeida JO. Autopercepção do estado de saúde: um estudo de prevalência com adolescentes de Ceilândia, Distrito Federal, Brasil. Comun Ciênc Saúde 2006;17(1):9-15.

20. Capilheira MF, da Silva Dos Santos I. [Individual factors associated with medical consultation by adults]. Rev Saude Publica 2006;40(3):436-43.

21. Da Silva MA, Rivera IR, De Souza MG, Carvalho AC. Medida da pressão arterial em crianças e adolescentes: recomendações das diretrizes de hipertensão arterial e prática médica atual. Arq Bras Cardiol 2007;88(4):491-5.

22. Goodwin DK, Knol LL, Eddy JM, Fitzhugh EC, Kendrick OW, Donahue RE. The relationship between self-rated health status and the overall quality of dietary intake of US adolescents. J Am Diet Assoc 2006;106(9):1450-3.

23. Neutzling MB, Araujo CL, Vieira Mde F, Hallal PC, Menezes AM. [Frequency of high-fat and low-fiber diets among adolescents]. Rev Saude Publica 2007;41(3):336-42.

24. Vancea LA, Barbosa JMV, Menezes AS, Santos CM, Barros MGV. Associação entre atividade física e percepção de saúde em adolescentes: revisão sistemática. Rev Bras Ativ Fis e Saúde 2011;16(3):246-254. 
25. Breidablik HJ, Meland E, Lydersen S. Self-rated health during adolescence: stability and predictors of change (Young-HUNT study, Norway). Eur J Public Health 2009;19(1):73-8.

26. Elinder LS, Sundblom E, Rosendahl KI. Low physical activity is a predictor of thinness and low self-rated health: gender differences in a Swedish cohort. J Adolesc Health 2011;48(5):481-6.

27. Gomes GA, Reis RS, Parra DC, Ribeiro I, Hino AA, Hallal PC, et al. Walking for leisure among adults from three Brazilian cities and its association with perceived environment attributes and personal factors. Int J Behav Nutr Phys Act 2011;8:111.

28. Galan I, Meseguer CM, Herruzo R, Rodriguez-Artalejo F. Self-rated health according to amount, intensity and duration of leisure time physical activity. Prev Med 2010;51(5):378-83.

29. Szklo AS, Coutinho ES. Vulnerability and self-perceived health status among light and heavy smokers: the relationship to short-term fear appeal tobacco control messages. Cad Saude Publica 2009;25(7):1534-42.

30. Meland E, Haugland S, Breidablik HJ. Body image and perceived health in adolescence. Health Educ Res 2007;22(3):342-50.

Endereço para Correspondência
Felipe Vogt Cureau
Rua João Rolim, n 418, Bairro Nossa
Senhora de Lourdes, CEP 97060-350,
Santa Maria, Rio Grande do Sul, Brasil
E-mail: f_c_vogt@yahoo.com.br

Recebido 27/11/2013

Revisado 27/12/2013

Aprovado 03/01/2014 